\title{
Linx
}

Revue des linguistes de l'université Paris X Nanterre

48 | 2003

Approches syntaxiques contemporaines

\section{Danielle Leeman, La phrase complexe. Les subordinations}

Ed. Duculot (coll. Champs linguistiques), 2002

\section{Christiane Marque-Pucheu}

\section{(2) OpenEdition}

\section{Journals}

Édition électronique

URL : http://journals.openedition.org/linx/232

DOI : $10.4000 /$ linx.232

ISSN : 2118-9692

\section{Éditeur}

Presses universitaires de Paris Nanterre

Édition imprimée

Date de publication : 1 juin 2003

Pagination : 161-163

ISBN : 0246-8743

ISSN : 0246-8743

\section{Référence électronique}

Christiane Marque-Pucheu, «Danielle Leeman, La phrase complexe. Les subordinations », Linx [En ligne], 48 | 2003, mis en ligne le 01 octobre 2003, consulté le 21 septembre 2020. URL : http:// journals.openedition.org/linx/232 ; DOI : https://doi.org/10.4000/linx.232 


\section{Comptes rendus}

\section{Compte rendu par Christiane Marque-Pucheu}

Danielle Leeman, La phrase complexe. Les subordinations, Ed. Duculot (coll. Champs linguistiques), 2002.

Si la subordination en français a été abordée dans tel ou tel chapitre de manuel ou fait l'objet d'un traitement à part entière dans des ouvrages de linguistique, aucun manuel n'y est spécifiquement dédié. Ce manque vient d'être comblé.

En cinq leçons encadrées par un sommaire et une table des matières détaillée, l'étudiant, qui n'est pas encore rompu au maniement des propriétés formelles, distributionnelles et transformationnelles des formes linguistiques et à leur interprétation, y est convié, tant sur des exemples élaborés à des fins didactiques que sur des extraits de documents authentiques. Car la démarche consiste à partir des formes, et, donc, à refuser l'intuition sémantique comme point de départ pour la description des unités syntaxiques. C'est seulement au terme de cette étape de traitement qu'il est possible de conclure au statut sémantique d'une unité donnée, une différence/similitude de forme pouvant être à l'origine d'une différence/similitude de sens. Dans cette conception saussurienne, la langue constitue un système autonome où la référence à la réalité n'est pas de mise. Puis des exercices sont proposés, parfois suscités par des articles ou des ouvrages de recherche ou de référence. Rien de mécanique dans ces exercices qui sollicitent non seulement la maitrise de la question, mais aussi un regard critique allié à la capacité d'argumenter. Assortis de corrigés comportant des conseils méthodologiques, ils sont suivis de suggestions de lectures.

Avant de définir la phrase complexe, D. Leeman définit la phrase simple (leçon 1) à partir de constituants qui l'organisent, ordonnés $G N+G V$. Elle refuse cette appellation aux suites n'entrant pas dans cette structure définitoire, notamment aux traditionnelles «phrases nominales». L'ensemble des deux types de suite reçoit l'appellation d'«énoncés ». Pour couper court à la confusion que pourrait introduire cet étiquetage, où « énoncé » n'est pas pris dans son acception coutumière, D. Leeman rappelle ce que la tradition grammaticale et linguistique entend habituellement par «énoncé ».

\footnotetext{
* La subordination infinitive a bien été traitée sous l'angle didactique (G. Legrand 1999), mais l'étude se limite à un article.
} 
Bien que deux leçons (2 et 3) y soient consacrées, la phrase complexe est abordée dès la première leçon : sous une forme apparemment identique (Paul va tomber et Eve espère réussir), une structure de phrase simple et une structure de phrase complexe sont respectivement mises en évidence par des propriétés discriminantes. Car il ne suffit pas d'avoir l'intuition grammaticale d'une différence de structure, il appartient aussi à l'étudiant de faire la démonstration de son savoir grammatical. Une très brève allusion est faite à la coordination dans Paul court et tombe, analysée comme opérant sur des phrases (sous-jacentes) : Paul court et Paul tombe. Les différents types de phrase complexe (juxtaposition, coordination, subordination) sont décrits également au début de la leçon 3 et le sujet se limite finalement, au sein de la phrase complexe, à la subordination.

Traitée dans les leçons 2 et 3 , elle est distinguée des autres types de phrase complexe par une «dépendance fonctionnelle ». Complétive, interrogative et relative (leçon 2) sont présentées successivement, puis les pièges liés, d'une part, à que conjonctif / que relatif et, d'autre part, aux différentes analyses de ce qui (ou ce que), sont déjoués. La subordination circonstancielle fait l'objet de la leçon 3. La notion de «circonstanciel» se dégage clairement, avec les réserves propres aux sous-classes de circonstanciels vis-à-vis des propriétés ; les différentes subordonnées circonstancielles ne se prêtent pas non plus de manière égale aux divers tests. Le traitement du mode rompt avec la conception traditionnelle qui associe à l'indicatif «faits réels » et au subjonctif « faits envisagés ».

La leçon 4 invite ensuite l'étudiant à réfléchir au triplet je vois que les enfants arrivent / je vois les enfants qui arrivent / je vois les enfants arriver. Si les tests révèlent facilement la différence d'analyse syntaxique entre complétive (voir a un cod) et infinitive (voir a deux compléments : un cod et un attribut de l'objet), la phrase avec relative est ambiguë, puisque cette dernière peut être épithète ou attribut. Des différences sémantiques séparent les trois constructions :

$>$ voir que n'est pas nécessairement un verbe de perception directe et peut inviter à une opération mentale (la question à quoi vois-tu cela? n'est acceptée que dans cette construction);

$>$ voir qui relève de la perception concrète, que celle-ci affecte l'entité désignée par le nom du GN objet (l'enfant qui est rentré tard hier soir, je le vois) ou l'événement asserté dans le contenu de la relative (Paul, je le vois qui avance);

$>$ voir suivi de l'infinitif peut, contrairement à la relative, traduire aussi un événement imaginaire (je vois Paul ne pas arriver).

La dernière leçon présente avec précision la théorie X-barre. Ses avantages sont soulignés : la représentation est particulièrement adéquate pour les phrases introduites par un que sans qu'il y ait pour autant subordination à une phrase. Les carences sont mentionnées, notamment la lourdeur «peu économique» de certaines analyses appliquées aux compléments de phrase pour rendre compte de leur mobilité ; de plus, la représentation à deux branches rend difficilement compte des cas où une tête (verbale) est associée à plusieurs compléments (direct et indirect, par exemple). Mais la théorie offre un caractère évolutif et des ajustements peuvent lui être apportés. 
Malgré un certain nombre de professions de foi, l'emprise de la pensée grammaticale traditionnelle persiste. C'est ainsi que le lecteur sera étonné de voir apparaitre en p. 91 l'expression d'« action verbale », vestige plus ou moins condamné en p. 10. La contestation de la tradition logico-grammaticale connait aussi parfois des imprécisions : ainsi, le développement intéressant sur la phrase et la proposition (p. 31) attribué à Wilmet ne doit l'être qu'en partie, car l'utilité du terme «proposition» avait déjà été remise en question dans Grammaire d'aujourd'bui.

L'idée d'une corrélation entre forme et sens exerce un certain pouvoir de séduction sur l'auteur et le lecteur : « là où la langue institue une différence de forme, alors (quelle que soit l'intuition) on doit découvrir une différence de sens, et de même, là où la langue institue une similitude de forme, doit pouvoir aussi se repérer une similitude sémantique. ». De même, on trouve quelques pages plus loin (p. 19) « .. du point de vue syntaxique, c'est la construction qui caractérise la modalité et non l'inverse, l'acte de langage qui caractérise la construction... ». Mais que dire alors du fait que la structure $\mathrm{GN}+\mathrm{GV}$ « ne correspond pas de manière univoque à l'intention déclarative... A l'inverse, une structure interrogative peut recouvrir une assertion. On n'a donc pas une relation biunivoque... »?

Dans la question 4 correspondant à la résolution de l'exercice 4 (leçon 1), il est dit : «Il y a tolère difficilement à sa suite un GN défini sans spécification ». Encore faudrait-il préciser qu'il convient de distinguer d'une part il y a existentiel, introduisant une propriété et associé à un GN indéfini uniquement (il y a un / *le problème qui est compliquê), d'autre part il y a présentatif, introduisant un événement et susceptible d'être associé à un GN défini (ily a un / le problème qui a tout bloquê).

Ces réserves n'entachent nullement la qualité linguistique et didactique du manuel. Le métalangage est réduit au minimum, n'entravant pas la lecture ; s'il y a lieu, le glossaire détaillé vient au secours de l'étudiant. Si un ouvrage précédent du même auteur ne portait pas le titre Méthodes en grammaire, celui-ci conviendrait parfaitement, tant les opérations syntaxiques sont bien expliquées et exploitées pour servir la démonstration. Le souci de l'étudiant se manifeste jusque dans le détail : souvent les éventuelles objections sont devancées, comme la possibilité d'observer une propriété dans un emploi d'une forme verbale, alors que la même propriété est interdite dans un autre. Mais surtout, il reçoit une leçon d'honnêteté, car il est convié à se frotter à différentes approches syntaxiques, traditionnelles ou autres, sans être inféodé à une école, l'objectif - atteint - étant l'initiation au raisonnement grammatical. Par exemple, la théorie guillaumienne oppose le mode du possible (subjonctif) au mode du certain et du probable (indicatif). De même, l'analyse distributionnelle est sollicitée (voir notamment la contrainte distributionnelle imposée au sujet des verbes admettant une complétive), mais lorsqu'elle s'avère inadéquate pour rendre compte des groupes prépositionnels dans Le père Noël a apporté un vélo à la selle large / à l'enfant sage / à l'heure dite, c'est l'analyse en constituants immédiats qui vient au secours de l'étudiant. Enfin, c'est une leçon de modestie, comme en témoignent ces mots : «la conclusion à laquelle on aboutit est susceptible d'être invalidée par des données auxquelles on n'a pas pensé jusqu'ici» (p. 51). 